\title{
Functional mitral regurgitation in chronic ischemic coronary artery disease: Analysis of geometric alterations of mitral apparatus with magnetic resonance imaging
}

\author{
$\mathrm{Hsi}-\mathrm{Yu} \mathrm{Yu}, \mathrm{MD}^{\mathrm{a}, \mathrm{c}}$ \\ Mao-Yuan Su, MS ${ }^{b}$ \\ Ta-Yu Liao, MS ${ }^{\mathrm{c}}$ \\ Hsu-Hsia Peng, MD ${ }^{d}$ \\ Fang-Yue Lin, $\mathrm{MD}, \mathrm{PhD}^{\mathrm{a}}$ \\ Wen-Yih Isaac Tseng, MD, PhD b,d
}

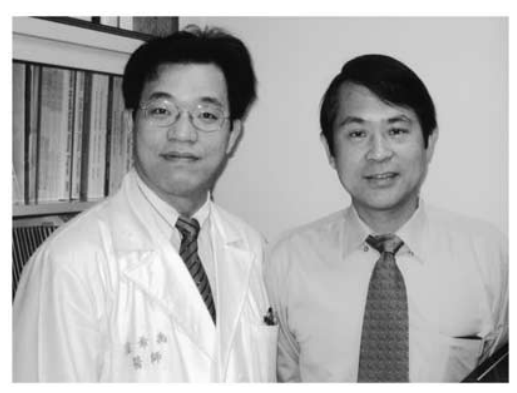

Dr Yu (left) and Dr Tseng (right)

From the Departments of Surgery ${ }^{\mathrm{a}}$ and

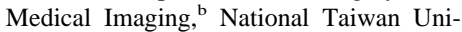
versity Hospital, and the Institute of Biomedical Engineering ${ }^{\mathrm{c}}$ and the Center for Optoelectronic Biomedicine, ${ }^{\mathrm{d}}$ National Taiwan University Medical College, Taipei, Taiwan, Republic of China.

This work was supported by the National Science Council, Taiwan (NSC91-2314-B002-217-M08 and NSC93WFA0100159).

Received for publication Dec 13, 2003; revisions received March 31, 2004; accepted for publication April 6, 2004

Address for reprints: Wen-Yih Isaac Tseng, MD, PhD, No. 1, Jen-Ai Road, Sec. 1, Center for Optoelectronic Biomedicine, National Taiwan University Medical College, Taipei, Taiwan, ROC (E-mail: wytseng@ha.mc.ntu.edu.tw).

J Thorac Cardiovasc Surg 2004;128:543-51 $0022-5223 / \$ 30.00$

Copyright ( $\odot 2004$ by The American Association for Thoracic Surgery

doi:10.1016/j.jtcvs.2004.04.015
Background: Patients with chronic coronary artery disease have double the mortality rate if the condition is combined with functional mitral regurgitation. An understanding based on geometric alterations of the mitral apparatus in functional mitral regurgitation is desirable.

Methods: Twenty-nine subjects were enrolled in the study, including 9 healthy volunteers (control group), 12 patients with chronic coronary artery disease without functional mitral regurgitation (CAD group), and 8 patients with chronic coronary artery disease with functional mitral regurgitation (CAD+FMR group). Cine magnetic resonance imaging was performed to acquire multiple short-axis cine images from base to apex. Left ventricular end-systolic volume, left ventricular ejection fraction, mitral area, and vertices of the mitral tetrahedron, defined by medial and lateral papillary muscle roots and anterior and posterior mitral annulus, were determined from reconstructed images at end-systole. Anterior-posterior annular distance, interpapillary distance, and annularpapillary distance (the distance from the anterior or posterior mitral annulus to the medial or lateral papillary muscle roots) were calculated.

Results: Left ventricular end-systolic volume was inversely associated with left ventricular ejection fraction $\left(R^{2}=0.778\right)$. Left ventricular end-systolic volume was highly associated with distances related to ventricular geometry $\left(R^{2}=0.742\right.$ for interpapillary distance, 0.792 for the distance from the anterior mitral annulus to the medial papillary muscle root, and 0.769 for distance from the anterior mitral annulus to the lateral papillary muscle root) but was moderately associated with distances related to annular geometry $\left(R^{2}=0.458\right.$ for anterior-posterior annular distance and 0.594 for mitral area, respectively). Moreover, interpapillary distance of greater than $32 \mathrm{~mm}$ and distance from the anterior mitral annulus to the medial papillary muscle root of greater than $64 \mathrm{~mm}$ readily distinguished the CAD+FMR group from the other groups.

Conclusion: In patients with coronary artery disease, an increase in left ventricular end-systolic volume is associated with inadequate approximation of the mitral tetrahedron during systole, which consequently leads to functional mitral regurgitation. Our study suggests that interpapillary distance and distance from the anterior mitral annulus to the medial papillary muscle root are sensitive to the increase in left ventricular end-systolic volume and reliably indicate the presence of functional mitral regurgitation.

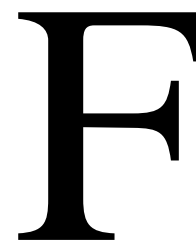

unctional mitral regurgitation (FMR) caused by ischemia is found in $20 \%$ of patients with chronic coronary artery disease (CAD) ${ }^{1}$ and in $59 \%$ of patients who present with poor left ventricular ejection fraction (LVEF). The mortality rate is doubled if chronic CAD is combined with FMR. Still, critical and effective treatments for FMR are lacking because of an inadequate understanding of the mechanism of this disease. In the present study geometric alterations of the mitral apparatus in 


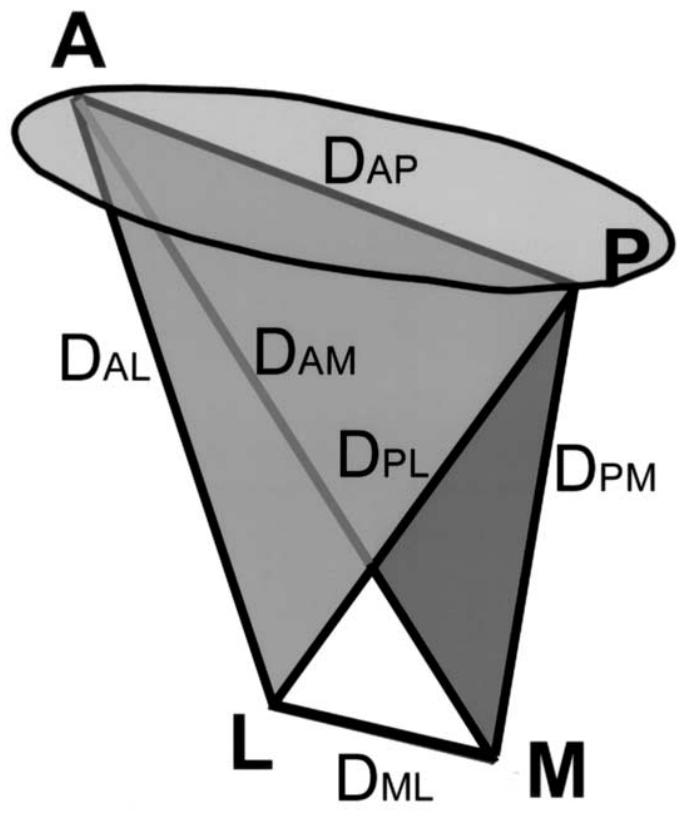

Figure 1. Illustration of the mitral area and mitral tetrahedron. The mitral tetrahedron was determined by 4 vertices $(A$, anterior annulus; $P$, posterior annulus; $M$, medial papillary root; $L$, lateral papillary root) and 6 edges $\left(D_{A M}\right.$, distance between the anterior annulus and the root of the medial papillary muscle; $D_{A L}$, distance between the anterior annulus and the root of the lateral papillary muscle; $D_{P M}$, distance between the posterior annulus and the root of the medial papillary muscle; $D_{P L}$, distance between the posterior annulus and the root of the lateral papillary muscle; $D_{A P}$, distance between the anterior annulus and the posterior annulus; $D_{M L}$, distance between the roots of the medial and lateral papillary muscle.)

patients with chronic CAD were characterized with magnetic resonance imaging (MRI). A mitral tetrahedron was defined by the medial and lateral papillary muscle roots and the anterior and posterior mitral annulus (Figure 1). The goal of the present study is to define the change in the mitral tetrahedron and its relationship with FMR.

\section{Methods}

\section{Study Population}

Twenty-nine subjects were enrolled in the study, including 9 healthy volunteers (control group), 12 patients with chronic CAD without FMR (CAD group), and 8 patients with chronic CAD with FMR (CAD+FMR group). Patients were given diagnoses of chronic CAD on the basis of positive findings on coronary angiograms, such as significant narrowing or tapering of the coronary arteries, and negative symptoms of acute coronary syndrome 1 month before MRI study. Mitral regurgitation was diagnosed by means of Doppler echocardiography with a semiquantitative scale on the basis of the presence of color jets occupying more than half of the left atrium and extending to the posterior portion of the atrium or into the left atrial appendage or pulmonary veins $(2+$ or more). The diagnosis of FMR was made after excluding obvious anatomic abnormalities of chordae or leaflets by using 2-dimensional echocardiographic examination. Left ventricular end-diastolic diameter (LVEDD), left ventricular end-systolic diameter (LVESD), and LVEF were determined by means of M-mode echocardiographic examination. Wall motion analysis was performed in a 2-dimensional mode. Location of old infarct was determined by means of Q-wave findings in ECG study. Age and sex distributions were comparable in the CAD and CAD + FMR groups, and body surface area was comparable in all 3 groups (Table 1). All subjects provided written informed consent before scanning. The study was carried out under the approval of the Institute Review Board of the National Taiwan University Hospital.

\section{Image Acquisition}

The study was performed in a 1.5T MRI system (Siemens Sonata). Cine MRI with a balanced steady state free precession sequence (TrueFISP, repetition time $[\mathrm{TR}]=30 \mathrm{~ms}$; echo time $[\mathrm{TE}]=1.5$ ms; flip angle $=60^{\circ}$; slice thickness $=10 \mathrm{~mm}$; field of view $[$ FOV $]=37 \times 30 \mathrm{~cm}$; matrix $=256 \times 208$ ) was acquired in the left ventricular short-axis planes from base to apex. The images were obtained under breath-holding conditions and with prospective ECG R-wave trigger mode. Approximately 8 to 12 short-axis slices were obtained, depending on the cardiac size (Figure 2). Setting temporal resolution at $30 \mathrm{~ms}$, the total number of cardiac phases was determined by dividing one effective R-R interval by $30 \mathrm{~ms}$. Heart rate was recorded at the beginning, middle, and end of the examination.

\section{Image Analysis}

End-systolic phase was identified as the time when the left ventricle showed maximal contraction, usually at 240 to $300 \mathrm{~ms}$ after the R-wave trigger. Multiple short-axis images from base to apex at this time point were used for 3-dimensional reconstruction. Left ventricular end-diastolic volume, left ventricular end-systolic volume (LVESV), and LVEF were derived with an in-house semiautomated algorithm reported previously. ${ }^{2}$ To reconstruct images in other planes, we interpolated the images into 60 to 80 layers in the short-axis view.

Three-dimensional coordinates of the anterior and posterior annulus and medial and lateral papillary muscle roots were determined in the reconstructed long-axis views (Figure 3 ). These 4 points defined the 4 vertices of a tetrahedron, representing the geometry of the mitral apparatus. The lengths of 6 edges of the mitral tetrahedron at end-systole were calculated (Figure 1).

Three-dimensional coordinates of points along the mitral annulus were selected from the reconstructed images. The size of the mitral area (MA) was calculated on the basis of the area enclosed by the selected points (Figure 3). All the image analysis was performed with Matlab (MathWorks Inc).

\section{Data Analysis}

Dichotomous data were compared by using the $\chi^{2}$ or Fisher exact tests. Numeric data were compared with the unpaired Student $t$ test. Test of correlation between different variables was performed with a linear regression model. All statistical works were performed with SPSS for Windows (SPSS Inc). 


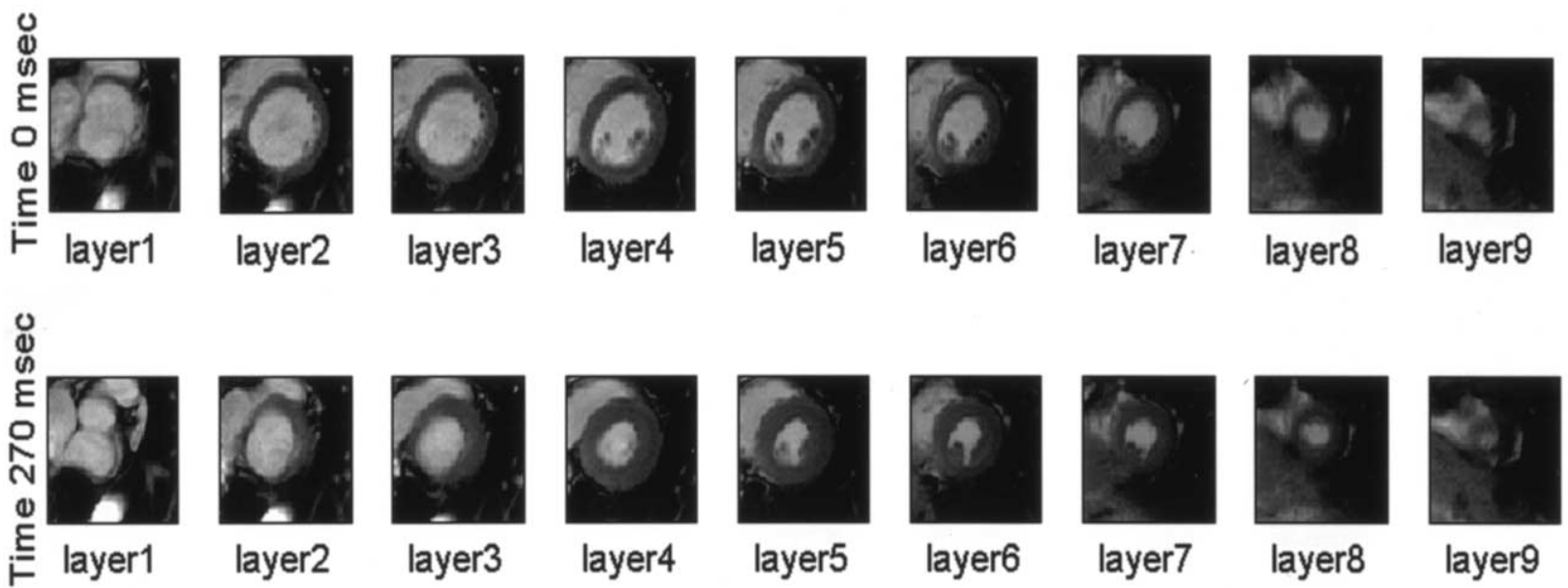

Figure 2. Cine MRI acquired in the left ventricular short-axis plane from base to apex. Gating acquisition was performed with prospective ECG R-wave triggers. Images at a temporal resolution of $\mathbf{3 0} \mathbf{m s}$ and in-plane spatial resolution of approximately $1 \times 1 \mathrm{~mm}^{2}$ were obtained. Cine images at end-diastole and end-systole are shown in the upper and lower rows, respectively.

TABLE 1. Demographic data of subjects

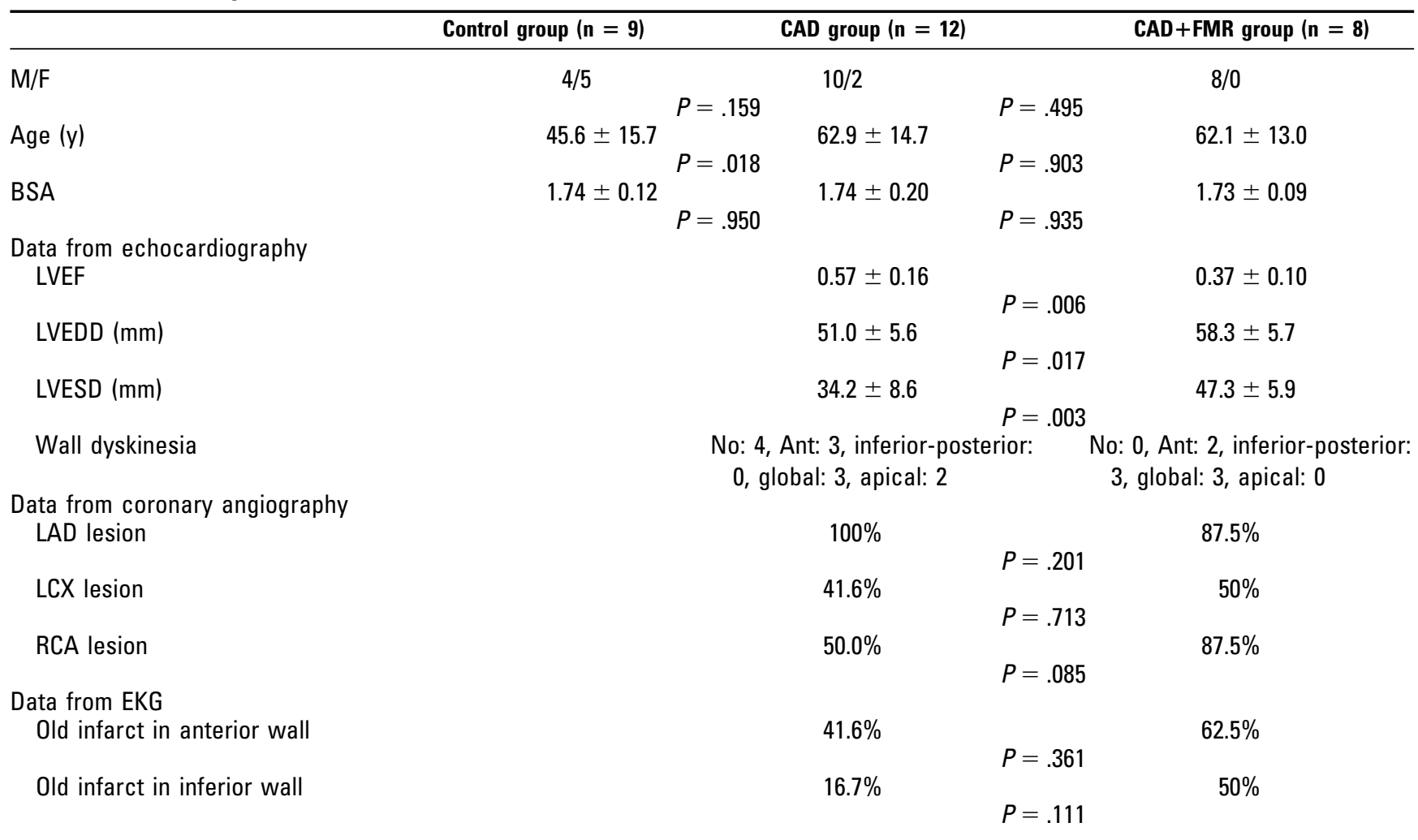

$P$ values for the comparison of control group versus CAD group and CAD group versus CAD FFM group are listed below each pair of data. $C A D$, Coronary artery disease; $C A D+F M R$, coronary artery disease with functional mitral regurgitation; $B S A$, body surface area; $L V E F$, left ventricular ejection fraction; $L V E D D$, left ventricular end-diastolic diameter; $L V E S D$, left ventricular end-systolic diameter; $L A D$, left anterior descending artery; $L C X$, left circumflex artery; $R C A$, right coronary artery; $E K G$, electrocardiography. 

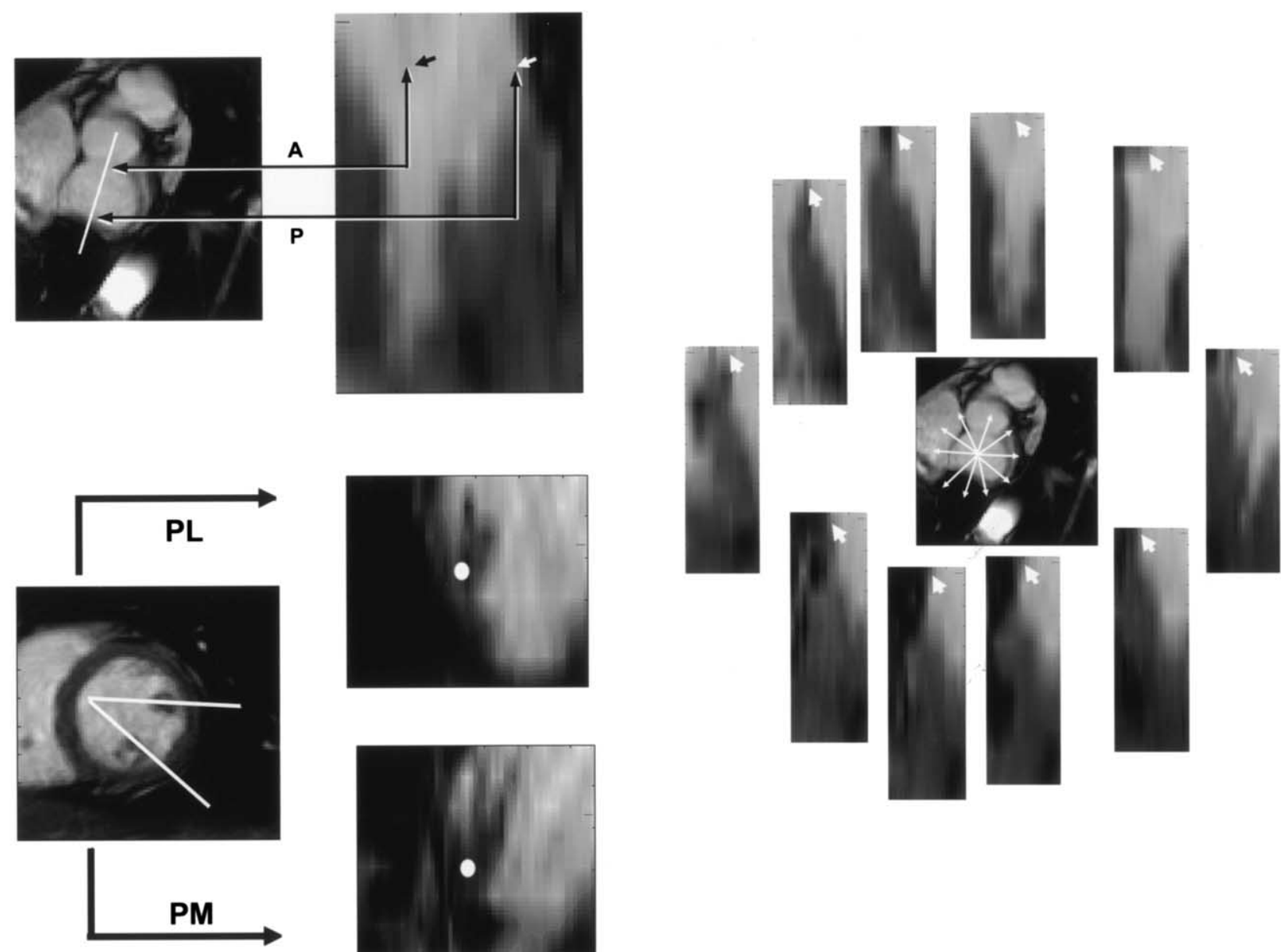

Figure 3. Interpolated and reconstructed images from original MRI data. Three-dimensional coordinates of the points of interest were determined on the long-axis planes decided from the lines of intersection on the short-axis plane. Left upper, Anterior annulus (denoted $A$ and indicated with black arrow on the right panel) and posterior annulus (denoted $\boldsymbol{P}$ and indicated with white arrow). Left lower, Roots of the medial papillary muscle and the lateral papillary muscle (indicated with white dots). Right, Ten points along the mitral annulus (indicated with arrowheads). PL, Root of lateral papillary muscle; PM, root of medial papillary muscle.

\section{Results}

In the control, CAD, and CAD+FMR groups LVESV was $29.1 \pm 17.0 \mathrm{~mL}, 60.3 \pm 38.4 \mathrm{~mL}$, and $183.4 \pm 76.7 \mathrm{~mL}$, respectively $(P=.023$ between the control and CAD groups and $P<.001$ between the CAD and CAD+FMR groups; Table 2), and LVEF was $0.65 \pm 0.11,0.50 \pm 0.19$, and 0.24 \pm 0.10 , respectively $(P=.048$ between the control and CAD groups and $P=.002$ between the CAD and CAD +FMR groups). On the basis of echocardiographic data, LVEF for the CAD +FMR group was less than that for the CAD group $(0.37 \pm 0.10$ vs $0.57 \pm 0.16, P=.006)$; LVEDD and LVESD for the CAD+FMR group was greater than that for the CAD group $(58.3 \pm 5.7 \mathrm{~mm}$ vs $51.0 \pm 5.6$ $\mathrm{mm}, P=.017$, for LVEDD; $47.3 \pm 5.9 \mathrm{~mm}$ vs $34.2 \pm 8.6$ $\mathrm{mm}, P=.003$, for LVESD). The CAD+FMR group was associated with a higher percentage of right coronary artery involvement and old inferior infarction than the CAD group (87.5\% vs $50.0 \%, P=.085$, for right coronary artery lesion; $50 \%$ vs $16.7 \%, P=.111$, for old inferior wall infarction).

In comparing edge lengths of the mitral tetrahedron between the control and CAD groups, MA and 5 of 6 edges of the mitral tetrahedron (distance between the anterior annulus and the root of the medial papillary muscle $\left[\mathrm{D}_{\mathrm{AM}}\right]$, distance between the anterior annulus and the root of the lateral papillary muscle $\left[\mathrm{D}_{\mathrm{AL}}\right]$, distance between the posterior annulus and the root of the medial papillary muscle $\left[D_{\mathrm{PM}}\right]$, distance between the posterior annulus and the root of the lateral papillary muscle $\left[\mathrm{D}_{\mathrm{PL}}\right]$, and distance between the anterior annulus and the posterior annulus $\left[\mathrm{D}_{\mathrm{AP}}\right]$ ) showed no significant differences between both groups, but the distance between the roots of the medial and lateral papillary muscle $\left(\mathrm{D}_{\mathrm{ML}}\right)$ was shorter in the control group 
TABLE 2. Left ventricular volumes, ejection fraction, edge lengths of the mitral tetrahedron and mitral area in different groups

\begin{tabular}{|c|c|c|c|c|c|}
\hline & Control group $(n=9)$ & & CAD group $(n=12)$ & & CAD+FMR group $(n=8)$ \\
\hline LVEDV (mL) & $80.6 \pm 31.9$ & & $109.0 \pm 39.3$ & & $234.3 \pm 69.8$ \\
\hline LVESV (mL) & $29.1 \pm 17.0$ & $\begin{array}{l}P=.092 \\
P=.023\end{array}$ & $60.3 \pm 38.4$ & $\begin{array}{l}P<.001 \\
P<.001\end{array}$ & $183.4 \pm 76.7$ \\
\hline LVEF & $0.65 \pm 0.11$ & $P=.048$ & $0.50 \pm 0.19$ & $P=.002$ & $0.24 \pm 0.10$ \\
\hline $\mathrm{D}_{\mathrm{AM}}(\mathrm{mm})$ & $50.9 \pm 7.8$ & $P=.559$ & $53.8 \pm 12.7$ & $P<.001$ & $80.5 \pm 8.6$ \\
\hline $\mathrm{D}_{\mathrm{AL}}(\mathrm{mm})$ & $48.6 \pm 4.5$ & $P=.841$ & $47.7 \pm 12.5$ & $P=.001$ & $71.1 \pm 12.4$ \\
\hline $\mathrm{D}_{\mathrm{PM}}(\mathrm{mm})$ & $46.8 \pm 8.3$ & $P=.544$ & $49.3 \pm 9.6$ & $P=.001$ & $65.5 \pm 6.4$ \\
\hline $\mathrm{D}_{\mathrm{PL}}(\mathrm{mm})$ & $45.7 \pm 7.1$ & $P=.880$ & $45.0 \pm 11.5$ & $P=.001$ & $63.8 \pm 8.6$ \\
\hline $\mathrm{D}_{\mathrm{ML}}(\mathrm{mm})$ & $14.8 \pm 5.9$ & $P=.013$ & $21.6 \pm 5.4$ & $P<.001$ & $40.0 \pm 6.3$ \\
\hline $\mathrm{D}_{\mathrm{AP}}(\mathrm{mm})$ & $30.4 \pm 3.8$ & $P=.352$ & $28.7 \pm 4.5$ & $P=.008$ & $45.6 \pm 13.4$ \\
\hline $\mathrm{MA}\left(\mathrm{cm}^{2}\right)$ & $9.24 \pm 2.52$ & $P=.431$ & $8.29 \pm 2.79$ & $P=.007$ & $18.6 \pm 7.80$ \\
\hline
\end{tabular}

$P$ values for the comparison between the control and CAD groups and between the CAD and CAD +FMR groups are listed below each pair of data. $C A D$, Coronary artery disease; $C A D+F M R$, coronary artery disease with functional mitral regurgitation; $L V E D V$, left ventricular end-diastolic volume; $L V E S V$, left ventricular end-systolic volume; $L V E F$, left ventricular ejection fraction. See Figure 1 for abbreviations of mitral tetrahedron.

than in the CAD group $(14.8 \pm 5.9 \mathrm{~mm}$ vs $21.6 \pm 5.4 \mathrm{~mm}$, $P=.013)$. In contrast, MA and all 6 edges of the mitral tetrahedron were longer in the CAD+FMR group than those in the CAD group (Table 2).

Regression analysis was performed to define the relationship between the geometry of the mitral tetrahedron and LVESV (Figure 4). LVEF was found to be inversely correlated with LVESV $\left(R^{2}=0.778\right)$. Different strengths of correlation were found between LVESV and the edge lengths of the mitral tetrahedron. Among the edge lengths in the longitudinal direction, the correlation was highest in $\mathrm{D}_{\mathrm{AM}}$ and $\mathrm{D}_{\mathrm{AL}}\left(R^{2}=0.792\right.$ and 0.769 , respectively $)$, followed by $\mathrm{D}_{\mathrm{PM}}$ and $\mathrm{D}_{\mathrm{PL}}\left(R^{2}=0.649\right.$ and 0.574 , respectively). In the transverse dimension, the correlation was high in $\mathrm{D}_{\mathrm{ML}}\left(R^{2}=0.742\right)$ but was moderate in $\mathrm{D}_{\mathrm{AP}}$ and MA $\left(R^{2}=0.458\right.$ and 0.594 , respectively).

The edge lengths of the mitral apparatus were further divided into 2 groups, one related to ventricular geometry, including $\mathrm{D}_{\mathrm{ML}}, \mathrm{D}_{\mathrm{AM}}, \mathrm{D}_{\mathrm{AL}}, \mathrm{D}_{\mathrm{PM}}$, and $\mathrm{D}_{\mathrm{PL}}$, and the other related to annular geometry, including $\mathrm{D}_{\mathrm{AP}}$ and MA. Compared with ventricular factors, a larger relative difference between the CAD+FMR and CAD groups was found in annular factors (Table 2). However, the variances of annular factors were even larger. Therefore the CAD +FMR group was more distinguishable from the other groups by using $\mathrm{D}_{\mathrm{ML}}$ and $\mathrm{D}_{\mathrm{AM}}$ as thresholds (32 and $64 \mathrm{~mm}$, respectively) but was less distinguishable if 2 annular factors, $\mathrm{D}_{\mathrm{AP}}$ and MA, were used (Figure 5).

\section{Discussion}

The present study, to our knowledge, is the first clinical study on the relationship of the mitral tetrahedron and FMR using MRI. Geometry of the mitral apparatus can be determined reliably with this method. Our study found that LVESV increased with a decrease in LVEF, and the increase of LVESV was associated with the increase of edge lengths of the mitral tetrahedron with variable strengths. Specifically, we found that $\mathrm{D}_{\mathrm{ML}}, \mathrm{D}_{\mathrm{AM}}$, and $\mathrm{D}_{\mathrm{AL}}$ showed strong association with LVESV $\left(R^{2}=0.742,0.792\right.$, and 0.769 , respectively) and that the CAD +FMR group was readily distinguishable from the other groups by using $\mathrm{D}_{\mathrm{ML}}$ and $\mathrm{D}_{\mathrm{AM}}$ as thresholds (32 and $64 \mathrm{~mm}$, respectively).

Ring annuloplasty is the most widely accepted surgical procedure for FMR in recent years. However, variable outcomes and a high rate of late recurrence have been reported. ${ }^{3}$ In addition, excessive downsizing of the circular ring can limit the dynamic motion of the mitral annulus or can cause complications of systolic anterior motion of the mitral anterior leaflet to obstruct the left ventricular outflow tract. ${ }^{4}$ The lack of effective treatment might be due to incomplete understanding of the pathophysiology of this complicated disease. Quantitative analysis of the mitral apparatus in patients with FMR, as presented in this study, provides information about geometric alteration of the apparatus during the process of FMR and helps clarify the effect of left ventricular dilation on each edge length of the mitral 


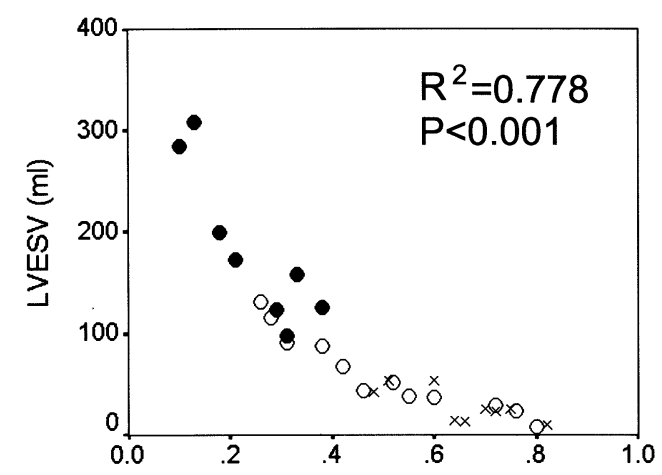

A

LVEF
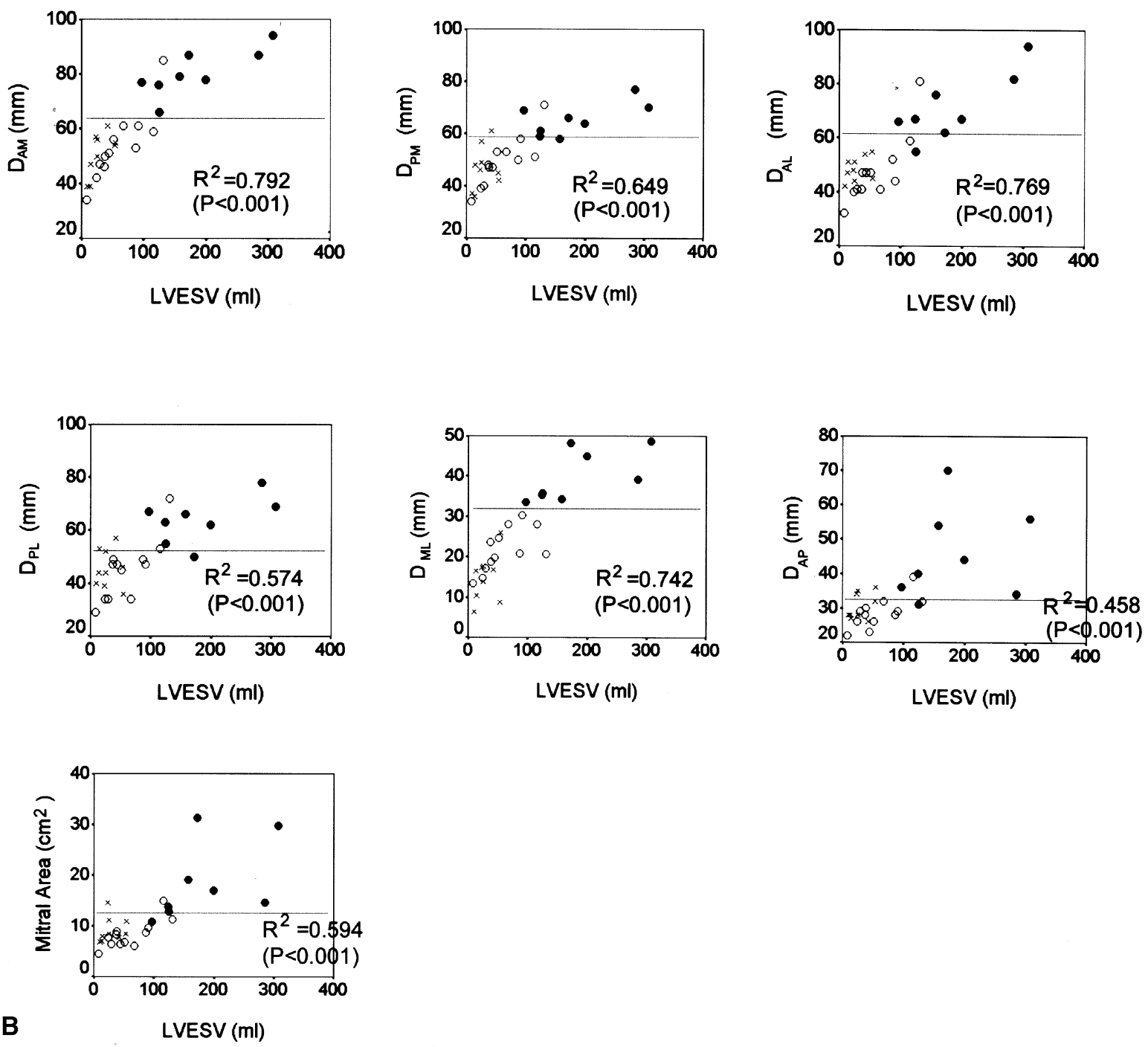

Figure 4. A, A scatter plot of the left ventricular end-systolic volume (LVESV) to the left ventricular ejection fraction (LVEF). B, Scatter plots of LVESV to each edge of the mitral tetrahedron and to the mitral area. $X$, Control group; open circle, CAD group; filled circle, CAD+FMR group. Horizontal lines indicate the cutoff values of respective parameters for FMR. 

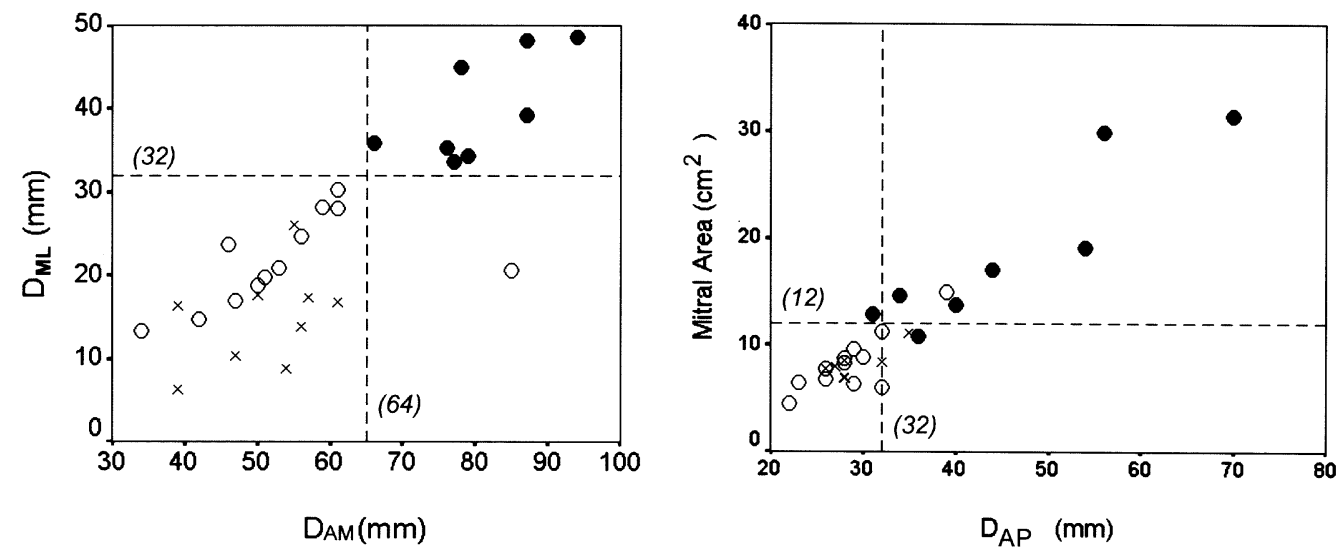

Figure 5. Scatter plots of 2 typical parameters of the ventricular factors $\left(D_{M L}\right.$ and $D_{A M}$, left panel) and the annular factors $\left(D_{A P}\right.$ and $M A$, right panel) for distinguishing the CAD+FMR group from the other groups. A clear distinction was found by using a $D_{M L}$ of $32 \mathrm{~mm}$ and a $D_{A M}$ of $64 \mathrm{~mm}$ as thresholds.

tetrahedron. On the basis of this knowledge, appropriate treatment methods could be developed.

Several study methods were used to investigate the pathophysiology of FMR, such as sonomicrometry crystal ${ }^{5}$ and radio-opaque marker placement ${ }^{6-9}$ for animal studies and echocardiography for animal or human studies. ${ }^{10}$ Standard echocardiography can only retrieve 2-dimensional images, and it is difficult to translate that data into 3-dimensional information. Assessment of the mitral apparatus by means of 3-dimensional echocardiography has been reported recently, ${ }^{11-13}$ but this method is time consuming and is not available for routine clinical use. ${ }^{14}$

Recent advance of the balanced steady state free precession sequence for cine MRI has shown the advantages of short acquisition time and good image quality. ${ }^{2}$ For each slice location, it takes about 12 seconds to acquire cine MRI of approximately 30 time frames. It can be readily achieved in a breath hold, effectively suppressing the artifacts from respiratory artifacts. It usually requires 8 to 12 slices to cover the whole left ventricle. The total scan time was less than 10 minutes. All our patients could lie still in the magnet and performed multiple breath holds throughout the study. The only obvious contraindication is arrhythmia, which will degrade the image quality and make interpretation difficult. We did not encounter this situation in our subjects.

Many factors were proposed to account for FMR, such as dilation and sphericalization of the left ventricle, ${ }^{15,16}$ altered leaflet tethering geometry, ${ }^{11}$ annular dilation, ${ }^{17}$ and papillary muscle discoordination. ${ }^{18}$ Our study shows that ventricular factors, namely $\mathrm{D}_{\mathrm{ML}}, \mathrm{D}_{\mathrm{AM}}, \mathrm{D}_{\mathrm{AL}}, \mathrm{D}_{\mathrm{PM}}$, and $\mathrm{D}_{\mathrm{PL}}$, have stronger association with the LVESV than annular factors, namely, $\mathrm{D}_{\mathrm{AP}}$ and MA (Figure 4, B). The lengthening of ventricular factors in systole can be attributed to general dilation of the left ventricle or to regional dyskinesia of the posterior wall. Among the ventricular factors, $D_{M L}$ and $\mathrm{D}_{\mathrm{AM}}$ are the most significant. All patients with FMR exclusively present with $D_{M L}$ values of greater than $32 \mathrm{~mm}$ and $\mathrm{D}_{\mathrm{AM}}$ values of greater than $64 \mathrm{~mm}$, suggesting that these 2 edge lengths might be further lengthened during the process of FMR. If proved, these 2 parameters might serve as indicators for the assessment and treatment of FMR.

Our results showed that LVESV increased as LVEF decreased. This finding is compatible with the previous reports that left ventricular dilation occurs as an early response of decreased LVEF that is mandated to generate a normal stroke volume from a large ventricular end-diastolic volume. ${ }^{19}$ Because of strong association $\left(R^{2}=0.742\right)$, we speculate that $D_{M L}$ is sensitive to the increase of LVESV. The increase of $\mathrm{D}_{\mathrm{ML}}$ theoretically pulls down the midpoints of the mitral leaflets (Figure 6, A), causing a predisposition toward central leakage. When the left ventricle dilates, its morphology changes from an ellipsoid to a spherical shape. ${ }^{15,16}$ The increase of the width and diagonal of left ventricular geometry is disproportionately greater than the increase of the height. This explains our finding that the association between LVESV and $\mathrm{D}_{\mathrm{AL}}$ or $\mathrm{D}_{\mathrm{AM}}$, representing the diagonal of the left ventricular geometry, was greater than that between LVESV and $\mathrm{D}_{\mathrm{PL}}$ or $\mathrm{D}_{\mathrm{PM}}$, representing the height of the left ventricular geometry (Figure $4, B$ ). In addition, the medial papillary muscle contributes more significantly than the lateral papillary muscle to the pathogenesis of FMR . 9,20,21 Therefore $\mathrm{D}_{\mathrm{AM}}$ was found to have the highest correlation with LVESV. The increase of annularpapillary distance tethers the mitral leaflets (Figure 6, $B$ ), and if the tethering effect hinders the closure of the mitral leaflets, FMR occurs.

Our study showed moderate correlation with LVESV in $\mathrm{D}_{\mathrm{AP}}$ and MA ( $R^{2}=0.458$ and 0.594 , respectively), indicating that the shape of the left ventricle in systole in some cases was more spherical, whereas that in others was more 
(A)

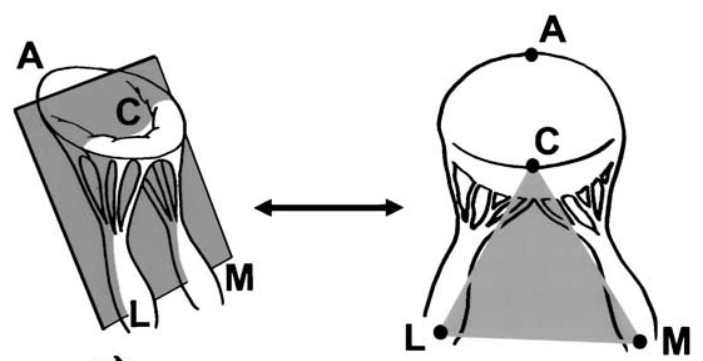

(B)

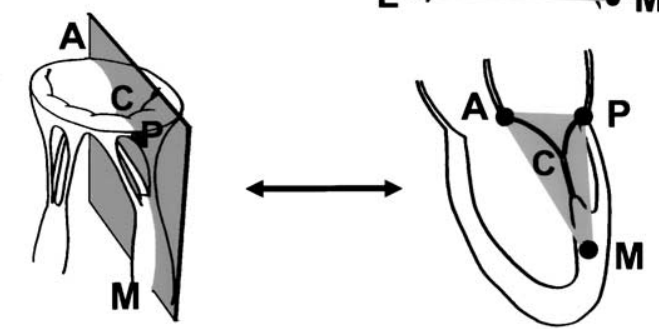

Figure 6. Illustration of the tethering effect by the structure of the mitral tetrahedron on the pathogenesis of FMR. A, Increased interpapillary muscle distance $\left(D_{\mathrm{ML}}\right)$ causes downward tethering of the midpoints of the mitral leaflets, a predisposition toward central leakage. B, When the increased annular-papillary distances are combined with increased anterior-posterior annular distance, the coapting points of the anterior and posterior leaflets (point $C$ ) were pulled apart. This causes FMR to happen.

ellipsoid. This implies that there might be different factors affecting the contractility of the annulus and mid ventricle, and these factors might have an effect on FMR.

To validate the data derived from MRI, we compared the results of the present study with those from animal studies using 3-dimensional echocardiography. ${ }^{13,22}$ The body weight, LVESV, and MA of the control group in the experimental dogs were 20 to $28 \mathrm{~kg}, 17.1 \pm 3.8 \mathrm{~mL}$, and $5.5 \pm$ $1.0 \mathrm{~cm}^{2}$, and those of control group in the experimental sheep were 40 to $50 \mathrm{~kg}, 19.5 \pm 1.6 \mathrm{~mL}$, and $6.1 \pm 0.3 \mathrm{~cm}^{2}$, respectively. These data were in proportion to the data of the present study in which the body weight, LVESV, and MA in the control group were 50 to $76 \mathrm{~kg}, 29.1 \pm 17.0 \mathrm{~mL}$, and 9.2 $\pm 2.5 \mathrm{~cm}^{2}$, respectively. One difference of the methodology in the present study from those seen in previous studies ${ }^{13,22}$ is that papillary muscle roots, instead of the papillary muscle tips, were used as one end of the annular-papillary distance. This is because the papillary muscle tips usually branch (Figure 3), and therefore the bias of estimation can be reduced if the papillary muscle roots are measured.

Theoretically, revascularization of affected myocardium (usually the posterior wall of the left ventricle) is an ideal way to treat ischemic FMR. But for many cases, the chronic ischemic myocardium has become fibrotic scar and cannot be vitalized by means of revascularization. Therefore surgical correction for FMR on the basis of geometric consideration might play its role in these cases. Up to now, several novel surgical methods, focusing on either annular or subvalvular components, ${ }^{8}$ were developed to correct for FMR. The methods include ring annuloplasty, Paneth suture annuloplasty, ${ }^{23}$ cutting of the basal chords to relieve leaflet retraction, ${ }^{24}$ use of a ventricular containment device to restrain left ventricular dilation, ${ }^{25}$ imbrication of interpapillary myocardium during ventricular restoration operation, ${ }^{26}$ and use of a papillary sling to bring both papillary muscles into close contact. ${ }^{27}$ Poor LVEF was often considered a risk factor for late recurrence of FMR in patients after ring annuloplasty. ${ }^{3,28}$ We speculate that this might be related to annular-papillary distances that increase with LVESV and inversely with LVEF. As shown in our study, increased annular-papillary distances of $\mathrm{D}_{\mathrm{ML}}$ and $\mathrm{D}_{\mathrm{AM}}$ were found exclusively in the CAD +FMR group. However, this increase is not corrected for by the procedure of ring annuloplasty.

According to the findings of the present study, we propose that FMR can be treated more effectively by means of 2 alternative approaches: first, cephalic mobilization of the papillary muscle roots to decrease annular-papillary distances in systole, thus reducing the tethering effect on the mitral leaflets, and second, reduction of the anterior-posterior annular distance to restore the reserve of coaptation between both leaflets combined with reduction of interpapillary distance to relieve tethering on the midpoint of leaflets, thus preventing the corresponding central leakage. The first approach might be achieved by using a ventricular containment device, and the second approach by using ring annuloplasty plus either a papillary sling or imbrication of the interpapillary myocardium.

\section{Limitations}

The present study focused on a group of patients with chronic CAD disease. Whether the derived conclusion applies to FMR in the acute condition or other diseases requires further study. The differences of parameters between the $\mathrm{CAD}$ and $\mathrm{CAD}+\mathrm{MR}$ groups can either be the cause or the results of FMR. For example, ventricular dilation can cause tethering of the mitral leaflets and FMR (type 3b). On the other hand, FMR can cause volume overload in the left ventricle and make it dilate. Our results suggest that, compared with annular factors, ventricular factors are more sensitive to the left ventricular dilation and that occurrence of FMR might aggravate the lengthening of $\mathrm{D}_{\mathrm{ML}}$ and $\mathrm{D}_{\mathrm{AM}}$. The interplay between LVESV, mitral tetrahedron, and FMR awaits further investigation by applying the present MRI method to a group of patients with acute or subacute FMR. The CAD group and the CAD + MR group were not matched in many parameters, including LVEF, LVESV, and left ventricular end-diastolic volume, which confound the search for a single parameter as the sole culprit of FMR. Further study on more matched groups is warranted to study 
a single factor leading to FMR (eg, any specific edge of the mitral tetrahedron or MA). However, from the surgical point of view, the parameters gathered from preoperative MRI, as presented in this study, can provide surgeons with a useful guide to determine which part of the mitral tetrahedron and how much of it should be downsized for correction.

Although the horizontal spatial resolution was high (inplane resolution of about $1 \mathrm{~mm}$ ), the longitudinal resolution was relatively low (slice thickness of $10 \mathrm{~mm}$ ) because of the requirement of reasonable examination time for the patients. Anatomic details, such as the mitral leaflets and chordae tendineae, cannot be delineated precisely in these images. The drawback of inadequate longitudinal resolution might compromise the accuracy of edge lengths in the longitudinal direction. Further development of faster cine MRI techniques or use of multidetector cardiac CT can provide more isotropic data for 3-dimensional reconstruction.

The effect of local ventricular function on papillary muscle motion and on annular contractility and their relationships to FMR were not investigated in this study.

\section{Conclusions}

This study introduces an MRI method to assess the geometry of the mitral apparatus in patients with chronic CAD with FMR. Alterations of edge lengths of the mitral tetrahedron can be determined for individual patients. According to the geometric information provided by using MRI, a surgical strategy can be custom designed.

We thank Dr Jaw-Lin Wang for helpful discussions and $\mathrm{Mr}$ Riley Nick for technical assistance.

\section{References}

1. Robbins JD, Maniar PB, Cotts W, Parker MA, Bonow RO, Gheorghiade $M$. Prevalence and severity of mitral regurgitation in chronic systolic heart failure. Am J Cardiol. 2003;91(3):360-2.

2. Tseng WY, Liao TY, Wang JL. Normal systolic and diastolic functions of the left ventricle and left atrium by cine magnetic resonance imaging. J Cardiovasc Magn Reson. 2002;4(4):443-57.

3. Tahta SA, Oury JH, Maxwell JM, Hiro SP, Duran CMG. Outcome after mitral valve repair for functional ischemic mitral regurgitation. J Heart Valve Dis. 2002;11(1):11-8.

4. Shah PM, Raney AA. Echocardiographic correlates of left ventricular outflow obstruction and systolic anterior motion following mitral valve repair. J Heart Valve Dis. 2001;10(3):302-6.

5. Gorman RC, Mccaughan JS, Ratcliffe MB, Gupta KB, Streicher JT, Ferrari VA, et al. Pathogenesis of acute ischemic mitral regurgitation in 3 dimensions. J Thorac Cardiovasc Surg. 1995;109(4):684-93.

6. Timek TA, Lai DT, Liang DH, Lo ST, Daughters GT, Ingels NB, et al. Timing and location of acute ischemic mitral regurgitation in sheep. Circulation. 2000;102(18):530-1.

7. Timek TA, Lai DT, Tibayan F, Liang D, Daughters GT, Dagum P, et al. Septal-lateral annular cinching abolishes acute ischemic mitral regurgitation. J Thorac Cardiovasc Surg. 2002;123(5):881-8.

8. Timek TA, Lai DT, Tibayan F, Liang D, Rodriguez F, Daughters GT, et al. Annular versus subvalvular approaches to acute ischemic mitral regurgitation. Circulation. 2002;106(suppl I):I27-32.

9. Tibayan FA, Rodriguez F, Zasio MK, Bailey L, Liang D, Daughters GT, et al. Geometric distortions of the mitral valvular-ventricular complex in chronic ischemic mitral regurgitation. Circulation. 2003; 108(suppl II):116-21.

10. Enriquez-Sarano R, Freeman WK, Tribouilloy CM, Orszulak TA, Khandheria BK, Seward JB, et al. Functional anatomy of mitral regurgitation-accuracy and outcome implications of transesophageal echocardiography. J Am Coll Cardiol. 1999;34(4):1129-36.

11. Otsuji Y, Handschumacher MD, Schwammenthal E, Jiang L, Song JK, Guerrero L, et al. Insights from three-dimensional echocardiography into the mechanism of functional mitral regurgitation-direct in vivo demonstration of altered leaflet tethering geometry. Circulation. 1997; 96(6):1999-2008.

12. Kaplan SR, Bashein G, Sheehan FH, Legget ME, Munt B, Li XN, et al. Three-dimensional echocardiographic assessment of annular shape changes in the normal and regurgitant mitral valve. Am Heart J. 2000;139(3):378-87.

13. Otsuji Y, Handschumacher MD, Liel-Cohen N, Tanabe H, Jiang L, Schwammenthal E, et al. Mechanism of ischemic mitral regurgitation with segmental left ventricular dysfunction: three-dimensional echocardiographic studies in models of acute and chronic progressive regurgitation. J Am Coll Cardiol. 2001;37(2):641-8.

14. Poutanen T, Jokinen E, Sairanen H, Tikanoja T. Left atrial and left ventricular function in healthy children and young adults assessed by three dimensional echocardiography. Heart. 2003;89(5):544-9.

15. Sabbah HN, Kono T, Rosman H, Jafri S, Stein PD, Goldstein S. Left-ventricular shape-a factor in the etiology of functional mitral regurgitation in heart-failure. Am Heart J. 1992;123(4):961-6.

16. Nass O, Rosman H, Alkhaled N, Shimoyama H, Alam M, Sabbah HN, et al. Relation of left-ventricular chamber shape in patients with low (less-than-or-equal-to-40-percent) ejection fraction to severity of functional mitral regurgitation. Am J Cardiol. 1995;76(5):402-4.

17. He SQ, Lemmon JD, Weston MW, Jensen MOJ, Levine RA, Yoganathan AP. Mitral valve compensation for annular dilatation: in vitro study into the mechanisms of functional mitral regurgitation with an adjustable annulus model. J Heart Valve Dis. 1999;8(3):294-302.

18. Gorman JH, Gorman RC, Jackson BM, Hiramatsu Y, Gikakis NS, Kelley ST, et al. Anterior and posterior papillary muscle discoordination, not annular dilatation, causes acute mitral regurgitation after posterior infarction. Circulation. 1996;94(8):3125.

19. Cohn JN. Structural basis for heart failure. Ventricular remodeling and its pharmacological inhibition. Circulation. 1995;91(10):2504-7.

20. Lai DT, Timek TA, Tibayan FA, Green GR, Daughters GT, Liang D, et al. The effects of mitral annuloplasty rings on mitral valve complex 3-D geometry during acute left ventricular ischemia. Eur J Cardiothorac Surg. 2002;22(5):808-16.

21. Komeda M, Glasson JR, Bolger AF, Daughters GT, MacIsaac A, Oesterle SN, et al. Geometric determinants of ischemic mitral regurgitation. Circulation. 1997;96(9):128-33.

22. Liel-Cohen N, Guerrero JL, Otsuji Y, Handschumacher MD, Rudski LG, Hunziker PR, et al. Design of a new surgical approach for ventricular remodeling to relieve ischemic mitral regurgitation: insights from 3-dimensional echocardiography. Circulation. 2000; 101(23):2756-63.

23. Tibayan FA, Rodriguez F, Liang D, Daughters GT, Ingels NB, Miller DC. Paneth suture annuloplasty abolishes acute ischemic mitral regurgitation but preserves annular and leaflet dynamics. Circulation. 2003; 108(10):128-33.

24. Messas E, Pouzet B, Touchot B, Guerrero JL, Vlahakes GJ, Desnos M, et al. Efficacy of chordal cutting to relieve chronic persistent ischemic mitral regurgitation. Circulation. 2003;108(10):111-5.

25. Sabbah HN. The cardiac support device and the myosplint: treating heart failure by targeting left ventricular size and shape. Ann Thorac Surg. 2003;75(suppl):S13-9.

26. Menicanti L, Di Donato M, Frigiola A, Buckberg G, Santambrogio C, Ranucci M, et al. Ischemic mitral regurgitation: intraventricular papillary muscle imbrication without mitral ring during left ventricular restoration. J Thorac Cardiovasc Surg. 2002;123(6):1041-50.

27. Hvass U, Tapia M, Baron F, Pouzet B, Shafy A. Papillary muscle sling: a new functional approach to mitral repair in patients with ischemic left ventricular dysfunction and functional mitral regurgitation. Ann Thorac Surg. 2003;75(3):809-11.

28. von Oppell UO, Stemmet F, Brink J, Commerford PJ, Heijke SA. Ischemic mitral valve repair surgery. $J$ Heart Valve Dis. 2000;9(1):64-73. 